\title{
Estudio de los procesos hidrológicos de la cuenca del Río Diguillín
}

\section{Study of the hydrological processes of the Río Diguillín watershed}

Fecha de entrega: 2 de marzo 2012

Fecha de aceptación: 24 de mayo 2012

\section{René Zúñiga ${ }^{1}$, Enrique Muñoz ${ }^{2}$ y José Luis Arumí ${ }^{3}$}

1 Moller y Pérez-Cotapos Construcciones Industriales S.A., Ezequías Alliende 2386, Providencia, Santiago, Chile, rzs@mollerci.cl 2 Departamento de Ingeniería Civil, Universidad Católica de la Santísima Concepción, Alonso de Ribera 2850, Concepción, Chile, emunozo@ucsc.cl

3 Departamento de Recursos Hídricos, Universidad de Concepción, Vicente Méndez 595, Chillán, Chile, jarumi@udec.cl

En el valle central del centro sur de Chile existe una gran demanda por recursos hidricos por parte de las actividades económicas como también por las demandas de una mejor calidad del ambiente. La agricultura es una de las principales actividades económicas de la zona, la cual requiere asegurar la disponibilidad de recursos con una planificación y gestión adecuada, en especial para escenarios hidro-meteorológicos que se alejan de las condiciones normales o medias. Para la gestión y planificación de recursos hídricos de la zona resulta necesario conocer los procesos hidrológicos que predominan en la generación de escorrentía y almacenamiento, y disponer de herramientas que permitan estimar condiciones futuras. En el presente estudio se implementa un modelo hidrológico sobre la cuenca del río Diguillín. El modelo incorpora una conexión entre el agua superficial y el agua subterránea en la zona alta de la cuenca con el objeto de reproducir el comportamiento de la cuenca de modo realista. El modelo una vez calibrado es capaz de reproducir condiciones pasadas. Luego, el modelo se utiliza para evaluar el comportamiento de la cuenca ante diferentes escenarios de variabilidad climática producidos por el fenómeno El Niño Oscilación del Sur.

Palabras claves: modelo de balance hídrico, disponibilidad y manejo de recursos hídricos, interacción agua superficial con agua subterránea, variabilidad climática
In the central valley in South-Central Chile there is a high demand for water resources from the different economical activities as well as from an increasing demand of better environment quality. Agriculture is among the main economic activities in this area, which requires ensuring the availability of water resources with a proper planning and management, especially for not normal or mean hydro-meteorological scenarios. For the planning and management of water resources it is necessary to understand the main hydrological processes that predominate in the runoff generation and storage, and to dispose of tools for the estimation of future conditions. In this study a hydrological model for the Rio Diguillin Watershed is implemented. The model incorporates a surface water and groundwater connection in the upper part of the watershed to reproduce by a realistic manner the observed behavior in the basin. Once the model is calibrated it is able to reproduce past conditions. Then, the model is used to evaluate the basin behavior under different scenarios of climate variability caused by El Niño Southern Oscillation.

Keywords: water balance model, water resources management and availability, surface-groundwater interaction, climate variability

disponibilidad de recursos hídricos, recursos que provienen principalmente de cuencas pre-andinas y andinas y donde su disponibilidad depende, en gran medida, de la capacidad de almacenamiento y regulación de estas cuencas y de la variabilidad climática que afecte la zona. En otras palabras, 
alteraciones y cambios en los patrones hidro-climáticos producidos por fenómenos regionales y globales, como El Niño Oscilación del Sur (ENOS) y cambio climático, pueden afectar directamente la hidrología de aquellas cuencas repercutiendo en la agricultura de riego. Por lo anterior, resulta necesario tener un conocimiento adecuado de las cuencas que almacenan y regulan los recursos hídricos en el valle central y de la hidrología de éstas, identificando procesos y sub-procesos predominantes en la disponibilidad hídrica.

Conocer e identificar los procesos predominantes en estas cuencas permite desarrollar y optimizar herramientas para la gestión y planificación de los recursos hídricos de modo de predecir el comportamiento hidrológico de una cuenca ante diferentes escenarios hidro-climáticos y de planificar y/o desarrollar planes de respuesta ante escenarios desfavorables.

Una alternativa para la gestión y administración de recursos hídricos es el uso de modelos hidrológicos. Los modelos hidrológicos han sido ampliamente usados en hidrología, siendo una herramienta útil para el entendimiento de los procesos relacionados con los recursos hídricos, para su cuantificación, para la estimación de impactos producto de los efectos del clima y para la estimación de los efectos por cambio de uso de suelo, entre otros.

Un modelo intenta reproducir bajo diferentes formas y procesos un fenómeno físico que ocurre sobre un objeto o territorio. Por lo tanto, en hidrología, un modelo busca representar un territorio delimitado por una divisoria de aguas (cuenca), y los fenómenos de transferencia de lluvia a caudal y de agua en el interior de ésta (Muñoz, 2011). En el presente trabajo se utilizó y modificó el modelo hidrológico presentado en Muñoz (2010) con el objeto de evaluar el comportamiento de la cuenca ante diferentes escenarios de variabilidad climática y de identificar procesos hidrológicos no simulados por dicho modelo en experiencias anteriores (presentadas en Böttcher, 2011). Böttcher (2011) obtuvo resultados no satisfactorios producto principalmente a que dicho modelo no fue capaz de simular procesos locales atribuibles a condiciones geológicas no consideradas.

\section{Área de estudio}

La Figura 1 muestra la cuenca del Río Diguillín. Ésta se sitúa en la ladera occidental de la cordillera de los Andes en el centro sur de Chile. La cuenca del Río Diguillín es una subcuenca de la del Río Itata y se ubica entre las latitudes $36^{\circ} 48^{\prime} \mathrm{S}, 37^{\circ} 03^{\prime} \mathrm{S}$ y longitudes $71^{\circ} 19^{\prime} \mathrm{W}, 72^{\circ} 22^{\prime} \mathrm{W}$. Posee un área total de $1257 \mathrm{~km}^{2}$ y su elevación varía entre 100 y $3175 \mathrm{msnm}$ en el límite oriental. Nace bajo el complejo volcánico Nevados de Chillán, desde donde nace el Estero Renegado (su principal tributario) y donde se origina el valle Nevados de Chillán. Por otra parte, se encuentra en una de las regiones extra-tropicales más afectadas por el ENOS (Grimm et al., 2000), presentando estacionalidad $\mathrm{y}$ variabilidad interanual en los patrones locales hidrometeorológicos (Grimm et al., 2000, Montecinos y Aceituno, 2003).

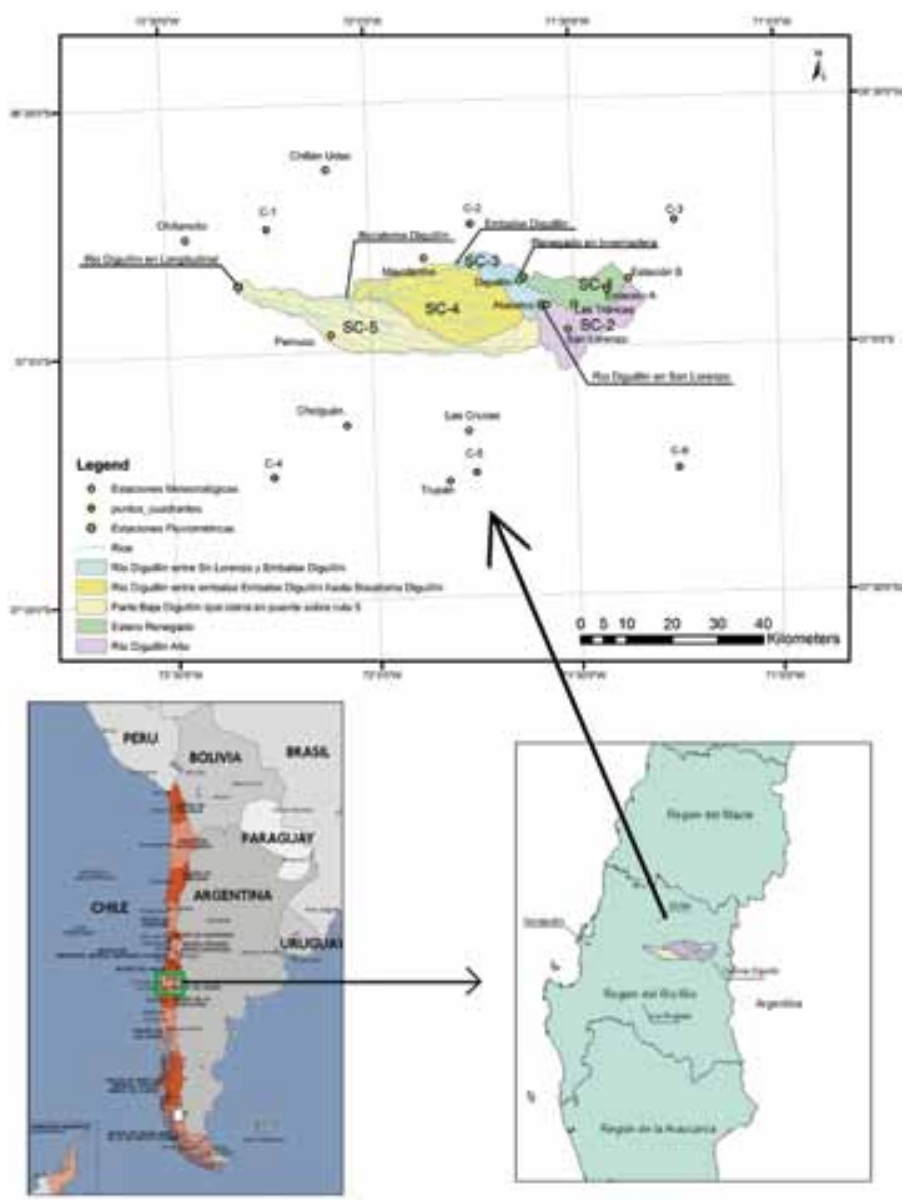

Figura 1: Mapa de ubicación de la cuenca del Río Diguillín

La precipitación media anual sobre la cuenca es de 1875 $\mathrm{mm}$ con un período pluvial durante el invierno y un período 
de deshielo y derretimiento de nieve durante la primavera e inicio del verano, mientras que la temperatura media mensual del aire en la cuenca es de $12.4^{\circ} \mathrm{C}$ con un rango entre $6^{\circ} \mathrm{C}$ en invierno a $20^{\circ} \mathrm{C}$ en verano.

Debido a la ubicación en la montaña de la cuenca, lo cual se refleja en su geología y morfología, la cuenca presenta alta variabilidad temporal con respecto a las características hidro-meteorológicas, donde el efecto orográfico produce un incremento en los montos de las precipitaciones (Garreaud, 2009, Vicuña et al., 2011, Dixon et al., 1999) y una conexión a nivel de cuenca y subcuenca de tipo interacción agua superficial-subterránea. Adicionalmente, esta es una zona afectada por el fenómeno ENOS. El ENOS es un fenómeno acoplado entre el océano y la atmósfera que se caracteriza por fluctuaciones irregulares (de 2 a 7 años de periodicidad), donde la alternancia de El Niño/La Niña son la principal fuente de variabilidad interanual. Los episodios de El Niño/La Niña están asociados a precipitaciones por sobre/bajo la media donde la temperatura del aire es más cálida/fría de lo normal (Garreaud, 2009).

Con la finalidad de estudiar los procesos hidrológicos a escala de subcuenca, la cuenca del Río Diguillín se subdividió en 5 subcuencas, las cuales se definieron según las zonas de interés a analizar y según la disponibilidad de estaciones de control para la posterior calibración y validación del modelo. La sub-división se hizo separando los tramos del Río Renegado en Invernada (SC-1), Río Diguillín en San Lorenzo (SC-2) y Río Diguillín Tramo Bajo (SC-3*), la cual se descompuso a su vez en Embalse Diguillín (SC-3), Bocatoma Diguillín (SC-4) y Río Diguillín en Longitudinal (SC-5). En la Figura 1 se muestra un esquema con la subdivisión por subcuencas antes mencionada.

\section{Descripción del modelo hidrológico}

En el presente estudio se utilizó el modelo de balance hídrico pluvio-nival y semi-distribuido de tipo conceptual presentado en Muñoz (2010) y Muñoz et al., (2011). El carácter conceptual del modelo puede ser discutible debido a que los modelos de los cuales se obtienen resultados numéricos son comúnmente designados como modelos de tipo matemáticos. Este modelo simula los procesos pluviales y de derretimiento de nieve por separado y permite incluir alteraciones antropogénicas sobre el régimen de caudales sumando o restando los flujos. La Figura 2 muestra un esquema del modelo utilizado.

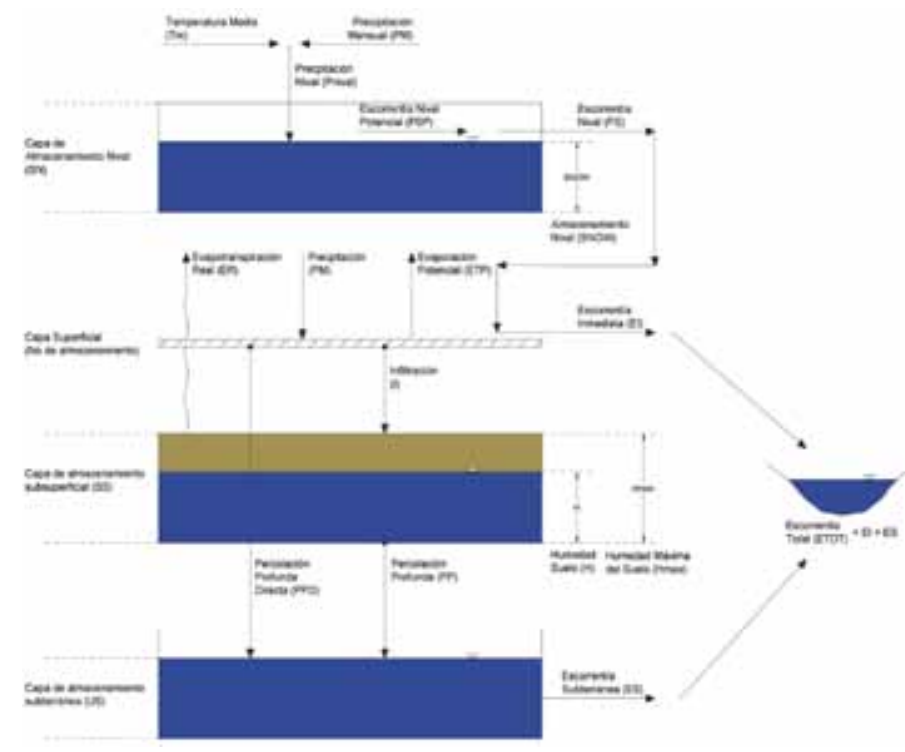

Figura 2: Diagrama descriptivo del modelo hidrológico (Muñoz, 2010)

La componente pluvial se modela a través de un modelo de precipitación-escorrentía que considera la cuenca como un sistema de doble almacenamiento, un sistema de almacenamiento superficial (SS) y un sistema de almacenamiento subterráneo (US). El SS representa el agua almacenada en el estrato de suelo no saturado, es decir, como humedad del suelo (H). El US representa el agua almacenada en el estrato de suelo saturado. El modelo requiere de dos variables de entrada, la precipitación (PM) y la evapotranspiración potencial (ETP). La salida del modelo es la escorrentía total (ETOT) a la salida de cuenca, la cual se compone de la suma de escorrentía subterránea (ES) más la escorrentía directa o superficial (EI). Los montos de escorrentía se calculan a través de seis parámetros de calibración, más dos parámetros que permiten analizar la sensibilidad de las variables de entrada PM y ETP.

El módulo nival determina la nieve caída $\left(\mathrm{P}_{\text {nival }}\right)$ basado en la precipitación por sobre la isoterma de $0^{\circ} \mathrm{C}$. Para ello, se estima una temperatura media representativa de la cuenca y se asume que ésta actúa sobre el centro de gravedad 
de la cuenca. Luego, utilizando la curva hipsométrica de la cuenca y el gradiente de temperatura de la zona de estudio, se determina dónde (a qué elevación) se tiene la isoterma cero, isoterma que de acuerdo al modelo separa la precipitación pluvial de la nival. Luego, $\mathrm{P}_{\text {nival }}$ es almacenada en un tercer sistema de almacenamiento que es el sistema de almacenamiento nival $\mathrm{SN}$, y así se obtienen los resultados de derretimiento basándose en el concepto del método de grados-día (Rango y Martinec, 1995). Utilizando el método de grados-día, se calcula el derretimiento potencial (PSP) y luego sobre la base de la nieve almacenada, se determina el derretimiento real (PS). Luego, PS se distribuye en el módulo pluvial a través de un parámetro de calibración. La Tabla 1 presenta una breve descripción de los parámetros y su influencia en el modelo.

Tabla 1: Parámetros del módulo pluvial y nival del modelo hidrológico mensual

\begin{tabular}{|c|c|c|c|}
\hline \multicolumn{2}{|c|}{ Parámetro } & \multirow{2}{*}{$\begin{array}{l}\text { Descripción } \\
\text { Coeficiente de escorrentía máxima cuando } \\
\text { el almacenamiento sub-superficial está } \\
\text { saturado }\end{array}$} & \multirow{2}{*}{$\begin{array}{l}\text { Influencia } \\
\text { EI }\end{array}$} \\
\hline \multirow{8}{*}{$\frac{\bar{\sigma}}{\grave{\Xi}}$} & $\mathrm{C}_{\text {máx }}$ & & \\
\hline & $\mathrm{P}_{\text {lim }}, \mathrm{mm}$ & $\begin{array}{l}\text { Monto de precipitación límite sobre el } \\
\text { cual existe percolación profunda directa } \\
\text { (PPD) }\end{array}$ & PPD \\
\hline & $\mathrm{D}$ & $\begin{array}{l}\text { Porcentaje de precipitación sobre } \mathrm{P}_{\lim } \text { que } \\
\text { se transforma en PPD }\end{array}$ & PPD \\
\hline & $\begin{array}{l}\mathrm{H}_{\text {máx }} \\
\text { mm }\end{array}$ & $\begin{array}{l}\text { Capacidad máxima de almacenamiento en } \\
\text { la Capa sub-superficial }\end{array}$ & $\mathrm{C}_{\text {máx }} \mathrm{y}$ ER \\
\hline & PORC & $\begin{array}{l}\text { Fracción de } \mathrm{H}_{\text {máx }} \text { que define el contenido } \\
\text { de agua en el suelo bajo el cual existen } \\
\text { restricciones sobre los procesos de } \\
\text { evaporación }\end{array}$ & $\mathrm{H}_{\text {crit }} \mathrm{y} \mathrm{ER}$ \\
\hline & $\mathrm{C}_{\mathrm{K}}$ & Coeficiente de escorrentía subterráneo & ES \\
\hline & A & $\begin{array}{l}\text { Factor de ajuste de los datos de } \\
\text { Precipitación }\end{array}$ & PM \\
\hline & B & \begin{tabular}{|l|}
$\begin{array}{l}\text { Factor de ajuste de los datos } \\
\text { evapotranspiración }\end{array}$ \\
\end{tabular} & PET y ER \\
\hline \multirow{5}{*}{$\stackrel{\bar{Z}}{\stackrel{\Xi}{Z}}$} & $\begin{array}{l}\mathrm{M}, \mathrm{mm} \\
{ }^{\circ} \mathrm{C}^{-1}\end{array}$ & $\begin{array}{l}\text { Fracción de nieve que se derrite sobre } \\
\text { una temperatura base }(\mathrm{Tb}) \text { de inicio del } \\
\text { derretimiento }\end{array}$ & PSP, PS \\
\hline & $\begin{array}{l}\mathrm{Tb} \\
{\left[{ }^{\circ} \mathrm{C}^{-1}\right]}\end{array}$ & $\begin{array}{l}\text { Temperatura base que indica el inicio del } \\
\text { derretimiento (normalmente } 0^{\circ} \mathrm{C} \text { ) }\end{array}$ & PSP, PS \\
\hline & $\mathrm{DM}$ & $\begin{array}{l}\text { Tasa mínima de derretimiento cuando Tm } \\
<\mathrm{Tb}\end{array}$ & PSP, PS \\
\hline & $\mathrm{F}$ & $\begin{array}{l}\text { Porcentaje de nieve derretida que se } \\
\text { incorpora a la escorrentía directa EI }\end{array}$ & EI \\
\hline & FgT & $\begin{array}{l}\text { Factor de ajuste de los datos de gradiente } \\
\text { térmico (debería ser } 1 \text { si el gradiente } \\
\text { térmico es medido en campo) }\end{array}$ & $P_{\text {nival }}$ \\
\hline
\end{tabular}

Por otra parte, el modelo comprende un módulo de alteraciones externas, el cual permite incorporar alteraciones al régimen como canales de trasvase, simulando la entrada y/o salidas hacia/desde una cuenca mediante la suma o resta de flujos según lo siguiente.

$\mathrm{Q}_{\text {out }}(\mathrm{t})=\operatorname{ETOT}(\mathrm{t})+\mathrm{Q}_{\text {contribuciones }}(\mathrm{t})-\mathrm{Q}_{\text {extracciones }}(\mathrm{t})$

Donde la descarga la cuenca $\mathrm{Q}_{\text {out }}$ en el paso de tiempo t, equivale a la escorrentía de la cuenca ETOT, más los caudales de aportes $\mathrm{Q}_{\text {contribuciones }}$ menos las extracciones $\mathrm{Q}_{\text {extracciones }}$ durante el mismo período. Para una descripción más detallada del modelo se recomienda revisar Muñoz (2010).

\section{Pre-proceso, datos de entrada y metodología}

Para el funcionamiento del modelo es necesario disponer de series de precipitación, temperatura del aire y evapotranspiración potencial, más la caracterización morfológica de la cuenca. Para la caracterización morfológica de la cuenca, se construyó un modelo digital de elevaciones utilizando datos de la Shuttle Radar Topography Mission (SRTM) de 3 arco-segundos (90 $\mathrm{m})$. A partir de la cual se obtuvieron descriptores morfométricos de la cuenca y sub-cuencas definidas.

En cuanto a los datos de entrada, se recolectaron series de precipitación de las estaciones pluviométricas Las Trancas, San Lorenzo, Las Cruces, Cholguán, Pemuco, Trupán, Chillancito, Diguillín, Atacalco (administradas por la Dirección General de Aguas, DGA), y Universidad de Concepción Campus Chillán (administrada por la Universidad de Concepción), y series sintéticas de temperatura publicadas por el Centro de Investigación sobre el Clima de la Universidad de Delaware UD (Willmot y Matsuura, 2008). Por otra parte, la evapotranspiración potencial se calculó utilizando el método de Thornthwaite y serie de datos de temperatura UD. La distribución espacial de dichas variables sobre la cuenca se realizó mediante polígonos de Thiessen.

Debido a la disponibilidad y calidad de los datos de entrada, el análisis se llevó a cabo a paso de tiempo mensual para un período de 22 años (1985 - 2008). La calibración y validación se realizó para el período 1985 -1998 y 1999 
- 2008 y sobre la base de tres sub-cuencas que disponen de control fluviométrico confiable, las SC-1, SC-2 y SC$3^{*}$ controladas por las estaciones Renegado en Invernada, Diguillín en San Lorenzo y Diguillín en Longitudinal respectivamente.

Cabe mencionar que con el objeto de mejorar la representatividad de los datos de entrada se crearon 2 estaciones pluviométricas con datos extrapolados de precipitaciones (Estaciones A y B, ver Figura 1). Puesto que la parte más alta de la cuenca (por sobre los 1200 $\mathrm{msnm}$ ) no posee registros pluviométricos y debido a que es necesario cuantificar el efecto orográfico presente en la zona, se crearon estaciones sintéticas correlacionando los montos de precipitación mensual con la elevación de las estaciones consideradas en el estudio. A partir de dicha correlación, se obtuvo un factor medio de 1.38 y 1.64 para la estación A y B respectivamente, donde dicho factor se aplicó sobre la última estación más arriba en la cuenca (Las Trancas).

Para llevar a cabo la calibración y validación del modelo, se utilizó la herramienta Monte Carlo Analysis Toolbox MCAT (Wagener y Kollat, 2007). MCAT es una herramienta que a través de simulaciones de Monte Carlo y que utilizando la metodología de estimación de incertidumbre probable generalizada (Beven y Binley, 1992) permite investigar la identificabilidad de un modelo y de sus parámetros. Esto es, la selección adecuada de un modelo, de su estructura y/o de los parámetros de éste a fin de reducir la incertidumbre de las salidas y de obtener un comportamiento realista o físicamente razonable de los procesos simulados por dicho modelo. MCAT permite calibrar un modelo de un modo semi-automático, permitiendo al usuario mantener un comportamiento identificable o realista del modelo. Para ello, MCAT opera ejecutando repetitivas simulaciones utilizando un set de parámetros seleccionados aleatoriamente dentro de un rango definido por el usuario. El programa almacena las salidas y los valores de la(s) función(es) objetivo (medida de la calidad de las simulaciones) para su posterior análisis.

Para la calibración se realizaron 15000 simulaciones utilizando rangos de los parámetros definidos a partir de experiencias previas (por ejemplo Muñoz, 2010; Ortiz et al., 2011; Muñoz et al., 2011; Böttcher, 2011) donde el propósito fue iterar utilizando conceptos de sensibilidad e identificabilidad (Wagener y Kollat, 2007) hasta encontrar el mínimo rango identificable de cada parámetro del modelo. Para ello se utilizaron gráficos de dispersión, los cuales relacionan el valor de cada parámetro con el valor de la función objetivo obtenido y gráficos de identificabilidad y sensibilidad regional, los cuales representan curvas de función de distribución acumulada (cdf) según una función objetivo pre-determinada. De 10 grupos de simulaciones (cada grupo contiene un $10 \%$ del total de simulaciones), el $10 \%$ de las mejores simulaciones es representado en una curva de color magenta, y el $10 \%$ de las peores simulaciones en una curva de color cian (Figura 4). Utilizando estas herramientas es posible determinar en qué rangos de cada parámetro, el modelo responde positiva o negativamente según los valores tomados por los parámetros. Esto permite identificar rangos acotados donde cada parámetro influye positivamente sobre la calidad de las simulaciones, lo cual se cuantifica a través de la función objetivo pre-definida.

La función objetivo utilizada fue el índice de eficiencia de Kling-Gupta KGE (Gupta et al., 2009).

$$
\mathrm{KGE}=1-\sqrt{(r-1)^{2}+(\alpha-1)^{2}+(\beta-1)^{2}}
$$

Donde $r$ es el coeficiente de correlación de Pearon, $\alpha$ es la razón entre la desviación estándar de los caudales simulados y observados, y $\beta$ es la razón entre los caudales medios simulados y observados. La función KGE es una mejora del índice de eficiencia de Nash-Suctliffe NSE (Nash y Sutcliffe, 1970), donde las componentes de correlación, desviación y variabilidad están ponderados de manera equitativa, resolviendo problemas sistemáticos de subestimación en los valores máximos y de poca variabilidad identificados en la función NSE (Gupta et al., 2009). Al igual que la función NSE, KGE varía entre - $\infty$ y 1 , siendo este último el valor óptimo. Para efectos del uso de MCAT se utilizó la transformada 1 - KGE, donde el valor óptimo es equivalente a 0 , y el peor modelo se asocia a $+\infty$. Si bien esta transformada no aporta en mayor medida al análisis, permite operar con MCAT puesto que por defecto MCAT asocia los valores óptimos de una función objetivo con los mínimos obtenidos de dicha función. 
Posterior a la calibración se realizó un proceso de validación donde se verificó que los parámetros de la mejor simulación presentaban resultados similares en la validación. Luego, con el objeto de evaluar diferentes escenarios de variabilidad climática (El Niño y/o La Niña) y de analizar la capacidad de respuesta y resiliencia de la cuenca ante dichos eventos, se realizaron las siguientes simulaciones en las 3 subcuencas utilizadas para la calibración del modelo: i) 5 años normales (año promedio), ii) 5 años secos (según año medio La Niña), iii) 5 años húmedos (según año medio El Niño), iv) 3 años secos y 2 años normales, v) 3 años húmedos y 2 años normales, vi) 3 años secos y 2 años húmedos, y vii) 3 años húmedos y 2 años secos. Resiliencia de la cuenca se interpreta como la capacidad de soportar eventos que difieren significativamente de las condiciones medias.

\section{Resultados y discusión}

En las Figuras 3 y 4 se muestran los gráficos de dispersión y de análisis de sensibilidad regional de los parámetros del modelo. Ambos gráficos corresponden a la primera iteración de la SC-1 considerando el rango inicial definido para los parámetros del modelo.
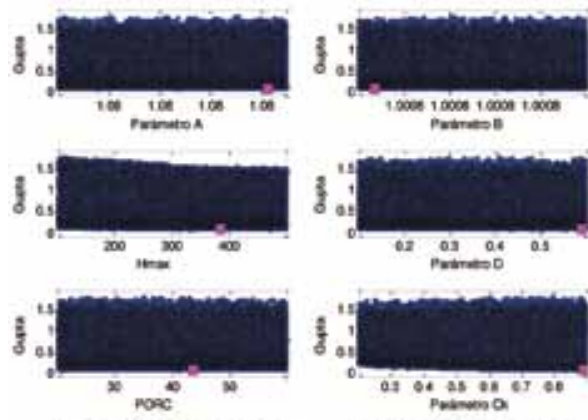

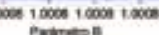

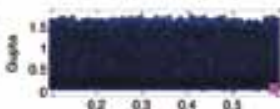
ox on at of
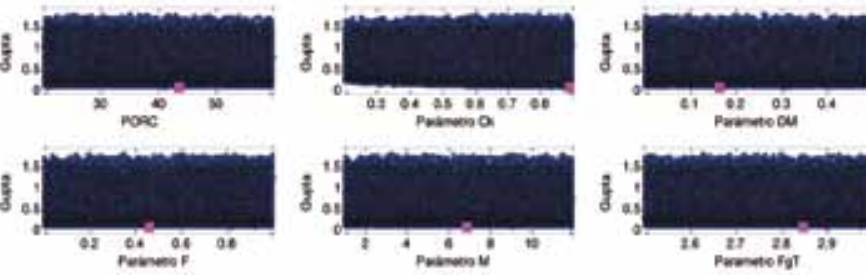

Figura 3: Diagrama de dispersión de parámetros del módulo pluvial y nival, considerando la función objetivo 1 - KGE para la primera iteración

Cabe mencionar que durante el proceso de calibración no se obtuvieron resultados aceptables, esto es, valores de KGE menores que 0.6. Por lo que en función de los resultados presentados por Böttcher (2011) donde se muestran diferencias superiores a un $100 \%$ entre caudales simulados y observados en el flujo base en las sub-cuencas
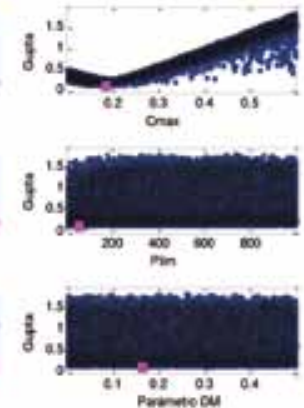

SC-1 y SC-2, y según afloramientos de aguas subterráneas observados en la ladera norte del Río Diguillín en el tramo superior (en la SC-2), se decidió modificar la estructura del modelo hidrológico incorporando una conexión de aguas subterráneas desde la SC-1 hacia la SC-2. El parámetro asociado a esta conexión que considera el porcentaje del flujo base que se transfiere de la SC-1 a la SC-2, el cual se determinó mediante un balance hídrico a largo plazo determinado para el período de calibración, y se verificó su representatividad para el período de validación. Dicha transferencia se estimó en un $77 \%$ del flujo base de la SC-1 que es aportado a la SC-2.
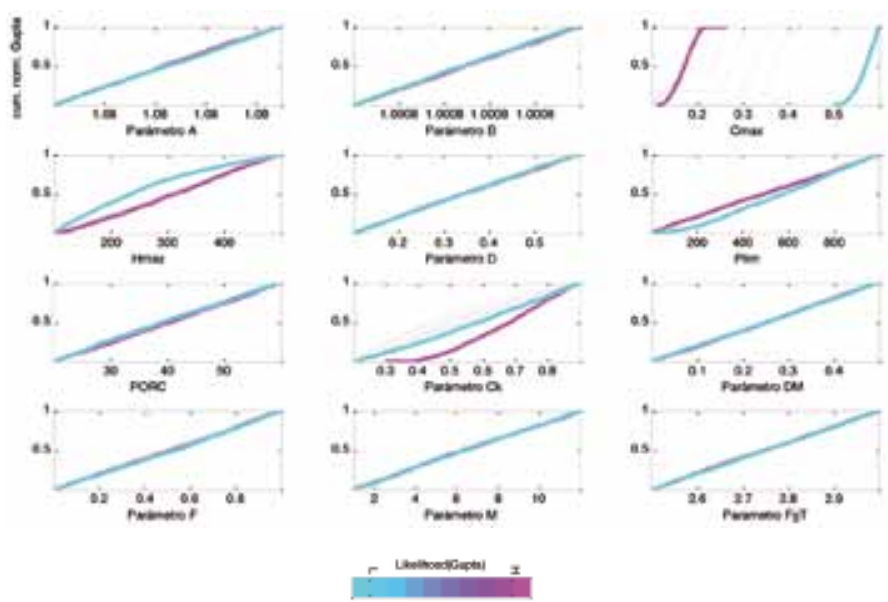

Figura 4: Análisis de sensibilidad e identificabilidad usando la función objetivo 1 - KGE, para la primera iteración

Del análisis se obtuvo que las sub-cuencas estudiadas presentaron sensibilidad e identificabilidad (mayor pendiente en cdf del $10 \%$ de las mejores simulaciones, curva color magenta) a los factores de modificación de las entradas A y B, y a los parámetros del modelo $\mathrm{C}_{\text {max }}, \mathrm{H}_{\max }$ $\mathrm{y}_{\mathrm{k}}$. Esto sugiere que los procesos predominantes en la hidrología de la cuenca del Río Diguillín son los procesos de escorrentía directa y flujo subterráneo, y el almacenamiento de agua en el suelo, el cual a su vez tiene relación directa con flujo subterráneo y con la evapotranspiración. Por otra parte, los parámetros asociados a la componente nival no presentaron mayor sensibilidad (curvas de pendiente constante en Figura 4), sugiriendo que dichos procesos son despreciables en dicha cuenca para el paso de tiempo mensual utilizado en el modelo.

En la Figura 3 se observa que el parámetro $C_{\max }$ posee 
un mínimo inferior a 0.2 , y en la Figura 4 se observa identificable entre 0.1 y 0.2 , valores que representan una cuenca con suelo de alta permeabilidad y pendientes fuertes, resultados similares a los observados en cuencas aledañas por Ortiz et al. (2011) y Muñoz et al. (2011). Por otra parte, los factores A y B fueron calibrados en 1.08 y 1 respectivamente para las $\mathrm{SC}-1$ y SC-3 con el fin de cerrar el balance hídrico a largo plazo. Lo anterior se traduce en que a pesar de extrapolar los montos de las precipitaciones en la parte alta de la cuenca, y de intentar incorporar en las series sintéticas el efecto orográfico, las series de precipitaciones de entrada de la SC-1 fueron subestimadas, por lo que tuvieron que ser amplificadas en $8 \%$ de modo de cerrar el balance hídrico a largo plazo, y mejorar la calidad de las salidas del modelo. De modo análogo, la serie de precipitaciones de la SC-3* tuvo que ser reducida en un $7 \%$ a fin de asegurar el cierre del balance hídrico a largo plazo de la sub-cuenca.

Por otro lado, en las Tablas 2 y 3 se muestran los parámetros del módulo pluvial y nival de las calibraciones de las 3 subcuencas. Donde los significados físicos de los parámetros tienen relación con los procesos e interacciones que ocurren en la cuenca.

Tabla 2: Valores finales de los parámetros del módulo pluvial, resultado de la calibración

\begin{tabular}{|l|r|r|r|}
\hline \multicolumn{4}{|c|}{ Módulo pluvial SC-1 SC-2 SC-3* } \\
\hline $\mathrm{C}_{\text {max }}$ & 0,17 & 0,6 & 0,6 \\
\hline $\mathrm{H}_{\max }, \mathrm{mm}$ & 322 & 200 & 229 \\
\hline $\mathrm{D}, \%$ & 31 & 16 & 28 \\
\hline $\mathrm{P}_{\text {lim }}, \mathrm{mm}$ & 50 & 29 & 390 \\
\hline PORC, \% & 20 & 35 & 29 \\
\hline $\mathrm{C}_{\mathrm{k}}$ & 0,89 & 0,86 & 0,9 \\
\hline $\mathrm{A}$ & 1,08 & 1,08 & 0,93 \\
\hline $\mathrm{B}$ & 1 & 1 & 1 \\
\hline
\end{tabular}

En la Tabla 4 se muestra el valor que adopta la función objetivo KGE, donde se observa que los valores de KGE más altos correspondes a las SC-2 y SC-3*, es decir, en aquellas subcuencas los caudales simulados se asemejan a los observados. Aunque la SC-1 no posee tan buenos resultados como las 2 subcuencas anteriores, los resultados se consideran aceptables.
Tabla 3: Valores finales de los parámetros del módulo nival, resultado de la calibración

\begin{tabular}{|l|r|r|}
\hline \multicolumn{3}{|c|}{ Módulo nival SC-1 SC-2 } \\
\hline $\mathrm{M}, \mathrm{mm}^{\circ} \mathrm{C}^{-1}$ & 5,3 & 1,9 \\
\hline $\mathrm{Tb},{ }^{\circ} \mathrm{C}$ & 0 & 0 \\
\hline $\mathrm{DM}$ & 0,42 & 0,39 \\
\hline $\mathrm{F}$ & 0,7 & 0,7 \\
\hline $\mathrm{FgT}$ & 2,7 & 2,9 \\
\hline
\end{tabular}

Tabla 4: Valores de la función objetivo para las diferentes subcuencas.

\begin{tabular}{|l|c|c|}
\hline KGE & Calibración & Validación \\
\hline SC-1 & 0,85 & 0,81 \\
\hline SC-2 & 0,92 & 0,91 \\
\hline SC-3* & 0,91 & 0,93 \\
\hline
\end{tabular}

En la Figura 5 se muestra los caudales simulados y los observados para la SC-1, para el periodo 1985-2008, donde se calibró entre 1985-1998 y se validó entre 19992008. De la figura se observa que los caudales simulados se asemejan a los observados, sin embargo, se observa entre los años 1989-1990, 1996-1997 y 2004-2005, los caudales simulados son mayores a los registrados. Éste se debe probablemente a la existencia de una conexión subterránea hacia la SC-3* no considerado en el modelo desarrollado.

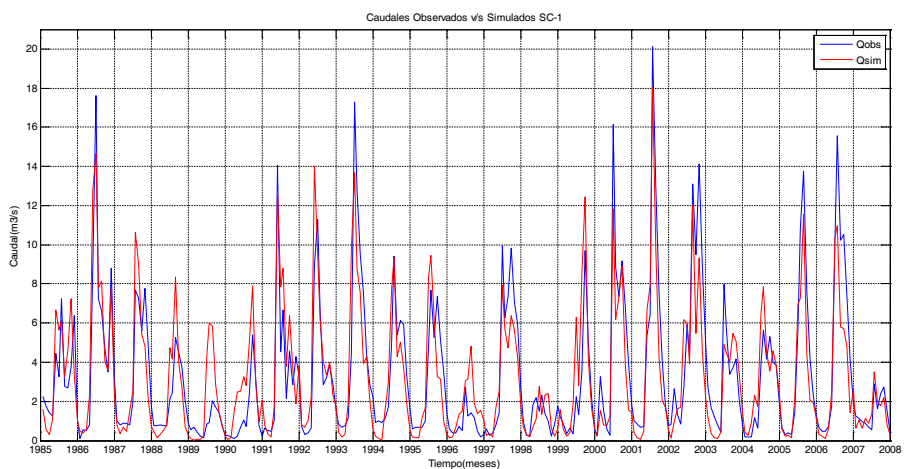

Figura 5: Comparación entre caudales simulados y observados para la SC-1. Períodos calibración y validación.

También se observa a principios de los años 1993-1996 que los caudales registrados son mayores a los simulados, esto se debe probablemente al derretimiento de glaciares que no están contempladas en el modelo. 
Para analizar la sensibilidad de la cuenca del Río Diguillín y de sus sub-cuencas ante escenarios de variabilidad climática se realizaron simulaciones a 5 años considerando combinaciones de años húmedos, secos y medios. En la Figura 6 se observa la conexión que existe entre el cambio en las precipitaciones y los caudales en la cuenca. Se observa que la escorrentía total es altamente sensible a la variabilidad de las precipitaciones. Además, se observa que el agua en la cuenca tiene un tiempo de residencia muy corto, ya que si se analiza el escenario de 3 años húmedos y luego 2 años secos, se observa que al pasar del año húmedo al seco, no existe acumulación ni liberación de agua de modo gradual ya que los flujos disminuyen rápidamente, asemejándose a la simulación generada para 5 años secos.

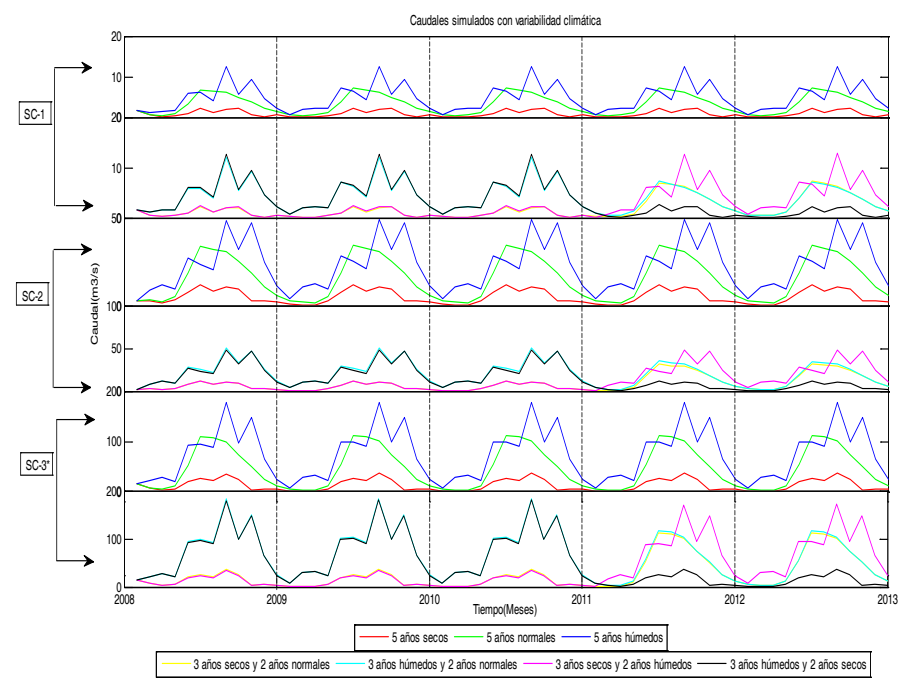

Figura 6: Simulaciones bajo escenarios de variabilidad climática para SC-1, SC-2 y SC-3*, en el periodo 2008-2013

Por otro lado, se observa que la cuenca tiene una baja capacidad de resistir periodos de sequía, ya que el caudal disminuye rápidamente a medida que también lo hacen las precipitaciones. Por el contrario, la cuenca posee una alta capacidad de recuperación, ya que el caudal aumenta rápidamente al pasar de un periodo seco a uno de condiciones normales o húmedos.

\section{Conclusiones}

Se identificaron los principales procesos hidrológicos generadores de escorrentía de la cuenca del Río Diguillín en diferentes zonas. A través de un modelo hidrológico se estableció una interacción de agua superficial con agua subterránea en el tramo superior de la cuenca, y una probable conexión entre las sub-cuencas del tramo superior con la zona baja y media del Río Diguillín. De esta forma el modelo es una herramienta de apoyo a la gestión y planificación adecuada de los recursos hídricos de la cuenca.

Se realizaron simulaciones para SC-3 y SC-4 con los parámetros del modelo validado, resultados que permiten apoyar la gestión de recursos de la zona pre-andina de la cuenca, donde se ubica el Embalse Diguillín y donde además se utiliza la mayor cantidad de recursos de la cuenca con fines agrícolas.

No se pudo cuantificar las recuperaciones en la parte baja de la cuenca atribuidas principalmente a exfiltraciones, ya que no se cuenta con puntos de medición adecuados ni suficientes, por lo cual se recomienda instalar más estaciones fluviométricas que puedan registrar caudales en la parte baja de la cuenca del Río Diguillín.

Se realizaron simulaciones de 5 años con una combinación de escenarios climáticos adversos para evaluar la vulnerabilidad debida a la variabilidad climática de la cuenca. Se encontró que la cuenca posee una baja capacidad de resistir periodos secos, ya que al disminuir las precipitaciones, el caudal disminuye rápidamente, sin existir un flujo que amortigüe estos cambios. Sin embargo, ésta posee una alta capacidad de recuperación, ya que al aumentar las precipitaciones aumenta rápidamente la escorrentía.

\section{Agradecimientos}

Los autores agradecen especialmente al Proyecto FONDECYT 1110298 Water availability in a stressed Andean watershed in central Chile: vulnerability Andean climate variability, por haber financiado parte importante de esta investigación.

\section{Referencias}

Beven, K. and Binley, A. (1992). The future of distributed models: model calibration and uncertainty prediction. Hydrological Processes 6, 279-298

Böttcher, W. (2011). Simulation of the precipitation-runoff behavior in the Diguillin Watershed (Central Chile). Diploma 
Thesis. Department of Physical Geography and Landscape Ecology, University of Leibniz Hannover, Germany

Dixon, H. J., Murphy, M.D., Sparks, S.J., Chávez, R., Naranjo, J.A., Dunkley, P.N., Young, S. R., Gilbert, J. S. and Pringle, M.R. (1999). The geology of Nevados de Chillán volcano, Chile. Revista Geológica de Chile 26(2), 227-253

Garreaud, R. D. (2009). The Andes climate and weather. Advances in Geosciences 22, 3-11

Grimm, A.M., Barros, V.R., and Doyle, M.E. (2000). Climate variability in southern South America associated with El Niño and La Niña events, Journal of Climate 13, 35-58

Gupta, H. V., Kling, H., Yilmaz, K. K. and Martinez, G. F. (2009). Decomposition of the mean squared error and NSE performance criteria: implications for improving hydrological modeling. Journal of Hydrology 377, 80-91

Montecinos, A. y Aceituno, P. (2003). Seasonality of the ENSOrelated rainfall variability in central Chile and associated circulation anomalies. Journal of Climate 16(2), 281-296

Muñoz, E., Álvarez, C., Billib, M., Arumí, J.L. and Rivera, D. (2011). Comparison of gridded and measured rainfall data for basin-scale hydrological studies, Chilean Journal of Agricultural Research71, N 3, 459-468

Muñoz, E. (2010). Desarrollo de un modelo hidrológico como herramienta de apoyo para la gestión del agua. Aplicación a la cuenca del río Laja, Chile. Tesis de Master, Departamento de Ciencias y Técnicas del Agua y del Medio Ambiente, Universidad de Cantabria, España

Muñoz, E. (2011). Perfeccionamiento de un modelo hidrológico aplicación de análisis de identificabilidad dinámico y uso de datos grillados. Tesis de Doctorado, Departamento de Recursos Hídricos, Universidad de Concepción, Chillán, Chile

Nash, J. and Sutcliffe, J. (1970). River flow forecasting through conceptual models part I: a discussion of principles. Journal of Hydrology 10(3), 282-290

Ortiz, G., Muñoz, E. y Tume, P. (2011). Incerteza en las variables de entrada de un modelo hidrológico conceptual. Efectos sobre la incerteza en las salidas. XX Congreso Chileno de Hidráulica, Octubre 2011, Santiago, Chile

Rango, A. and Martinec, J. (1995). Revisiting the degree-day method for snowmelt computations. Journal of the American Water Resources Association 31(4), 657-669
Vicuña, S., Garreaud, R. D., and McPhee, J. (2011). Climate change impacts on the hydrology of a snowmelt driven basin in semiarid Chile. Climatic Change 105: 469-488

Wagener, T. and Kollat, J. (2007). Numerical and visual evaluation of hydrological and environmental models using the Monte Carlo analysis toolbox. Environmental Modelling and Software 22, 1021-1033

Willmot, C. and Matsuura, K. (2008). Terrestrial air temperature and precipitation: Monthly and annual time series (1900-2008) Version 1.02. University of Delaware, http://climate.geog.udel. edu/climate 